\title{
Alterations in Fiber Pathways Reveal Brain Tumor Typology: a Diffusion Tractography Study
}

Conventional structural Magnetic Resonance (MR) techniques can accurately identify brain tumors but do not provide exhaustive information about the integrity of the surrounding/embedded white matter (WM). In this study, we used Diffusion-Weighted (DW) MRI tractography to explore tumor-induced alterations of WM architecture without any a priori knowledge about the fiber paths under consideration. We used deterministic multi-fiber tractography to analyze 16 cases of histologically classified brain tumors (meningioma, lowgrade glioma, high-grade glioma) to evaluate the integrity of WM bundles in the tumoral region, in relation to the contralateral unaffected hemisphere. Our new tractographic approach yielded measures of WM involvement which were strongly correlated with the histopathological features of the tumor $(r=0.83, p=0.0001)$. In particular, the number of affected fiber tracts were significantly $(p=0.0006)$ different among tumor types. Our method proposes a new application of diffusion tractography for the detection of tumor aggressiveness in those cases in which the lesion does not involve any major/known WM paths and when a priori information about the local fibers anatomy is lacking. 


\section{Alterations in Fiber Pathways Reveal Brain Tumor Typology: a Diffusion Tractography Study}

\section{Characterization of White Matter Damage}

Martina Campanella, PhD1,*, Tamara Ius, MD, PhD1,2, Miran Skrap, MD2 , Luciano Fadiga, MD, PhD1,3

1 Department of Robotics, Brain and Cognitive Sciences, Istituto Italiano di Tecnologia, Genoa, Italy

2 Department of Neurosurgery, Az. Ospedaliero-Universitaria Santa Maria della Misericordia, Udine, Italy 3 Section of Human Physiology, University of Ferrara, Italy

*Corresponding author:

Martina Campanella, $\mathrm{PhD}$

Department of Robotics, Brain and Cognitive Sciences

Istituto Italiano di Tecnologia

Via Morego 30, 16163, Genoa, Italy

Phone: +39010 71781420

Fax: +39010 717081

e-mail: martina.campanella@iit.it 


\section{Abstract}

20 Conventional structural Magnetic Resonance (MR) techniques can accurately identify brain 21 tumors but do not provide exhaustive information about the integrity of the 22 surrounding/embedded white matter (WM). In this study, we used Diffusion-Weighted (DW) 23 MRI tractography to explore tumor-induced alterations of WM architecture without any a priori 24 knowledge about the fiber paths under consideration. We used deterministic multi-fiber 25 tractography to analyze 16 cases of histologically classified brain tumors (meningioma, low26 grade glioma, high-grade glioma) to evaluate the integrity of WM bundles in the tumoral region, 27 in relation to the contralateral unaffected hemisphere. Our new tractographic approach yielded 28 measures of WM involvement which were strongly correlated with the histopathological features 29 of the tumor $(\mathrm{r}=0.83, \mathrm{p}=0.0001)$. In particular, the number of affected fiber tracts were 30 significantly $(\mathrm{p}=0.0006)$ different among tumor types. Our method proposes a new application of 31 diffusion tractography for the detection of tumor aggressiveness in those cases in which the

32 lesion does not involve any major/known WM paths and when a priori information about the 33 local fibers anatomy is lacking.

\section{Keywords: Brain Tumor, White Matter, Fiber Tractography.}


Introduction

A number of brain pathologies affect white matter (WM) fiber pathways, either by disruption, infiltration or displacement ${ }^{1,2}$. Knowledge of such alterations may provide useful information during neurosurgery, particularly in the case of infiltrating lesions, where the extent of excision and the prognosis are positively correlated ${ }^{3,4}$. In fact, while highly aggressive lesions significantly alter morphology and impair functionality of infiltrated WM, less aggressive tumors may simply displace the surrounding brain structures. These differing behaviors influence surgical strategy, mainly aimed at finding the best compromise between amount of tissue removed and degree of preservation of brain functionality.

NowAdays, advanced techniques such as diffusion tensor imaging (DTI) allow for noninvasive tracking of WM fiber bundles characterized by a well-known hodology and course (e.g. the corticospinal system). The task becomes more difficult, however, when a priori information about the anatomy of the local fibers is lacking.

DTI is based on the fact that water diffusion is greater along fiber main axis rather than perpendicularly to it and can be used to estimate fiber orientation in each MR voxel ${ }^{5}$. Tractography uses this local information from the reconstructed diffusion tensors to identify global white matter tracts ${ }^{6-8}$. Over the last decade, diffusion MRI has often been used to investigate WM alterations. Clinicians have gained useful insights from these studies for surgical planning $^{9,10}$ and assessing reorganization after injury ${ }^{11-12}$ or specific therapy ${ }^{13}$. Several reports have assessed damage to fiber tracts in relation to cerebral neoplasm types using DTI metrics, such as fractional anisotropy (FA) and mean diffusivities (MD) ${ }^{14-17}$. The majority of those works were based on a priori anatomical knowledge about fiber courses and use known anatomical landmarks to select seed regions and to reconstruct major fiber tracts ${ }^{18-22}$. 
However, WM mapping based on known normal anatomical locations as seeds might be

misleading in the presence of a tumor, since the WM architecture can be deviated from its normal location and edema can mask the path of fiber tracts. A complementary approach to increase precision is to integrate fMRI information ${ }^{23,24}$ or Intraoperative Electrical Stimulation (IES) ${ }^{25}$ data with tractographic data. IES is however invasive and can be performed only during surgery and not during surgical planning. Moreover, one must take into account the possible alteration of the functional responses (e.g. fMRI and IES responses) by the tumor ${ }^{26}$.

Since the diffusion tensor model infers the orientation of a single fiber per voxel, the DTI approach is only able to trace major tracts in the brain and cannot model complex WM architectures. Several approaches have recently been proposed to overcome this limitation and to estimate multiple fiber directions within the same voxel ${ }^{27-29}$. One of such technique, Persistent Angular Structure (PAS)-MRI ${ }^{30}$, starts from an economical spherical acquisition paradigm and computes a function of the sphere that reflects the angular structure of the water molecule displacement density by using the peaks of this function as fiber-orientation estimates. This particular method ensures high sensitivity to fiber crossings. Two families of tractography algorithms exist, deterministic ${ }^{31}$ and probabilistic ${ }^{32,33}$. In the latter, the range of probable fiber orientations is probed with a Monte Carlo strategy and the total number of fiber trajectories passing through each voxel provides a confidence level of that voxel inclusion in the tract of interest.

Here, we propose a novel approach assessing fiber displacement/disruption caused by brain lesions that is based on PAS-MRI deterministic tractography from HARDI data. Lesions are outlined (here for patients affected by histologically characterized gliomas or meningiomas) and mirrored onto the contralateral normal hemisphere. This new location was then used as seed region for PAS-MRI tractography of the HARDI data. The end point clusters of the tracts so 
82 obtained, as well as their back-reflection onto the affected hemisphere, are then used to generate a

83 further set of tracts back to the seed ROI (or the original lesion ROI). By this approach we 84 identify fiber bundles which would have presumably run through the tumor and their contralateral

counterparts for comparison. All possible distinct bundles and trajectories of tumor-involved WM tracts were evaluated in relation to the myeloarchitecture, without any atlas-guided tract reconstruction.

We evaluated the capability of our technique capability to determine the typology and aggressiveness of the lesion by analyzing the relationship between the severity of tumor-induced damage and lesion-specific histological features. In particular, we compared the alterations of fiber pathways between the two types of lesions considered, meningioma and gliomas. We also tested the method by characterizing the degree of WM alteration according to their MIB-1 labeling indexes ${ }^{34}$, a monoclonal antibody expression of the percentage of positive staining tumor cell nuclei. We used deterministic tractography via PAS-MRI as this is a straightforward, rapid technique. Alternatively, probabilistic tractography could be used, though the time demands are heavier and may not be compatible with routinary clinical use. In order to assess the impact of our choice, in the present study we compared our results with those of probabilistic tractography. This latter comparison showed our approach to yield tractography results similar to those of probabilistic tractography, whilst the tumor assessments showed significant differences in tractography results between the tumor subgroups studied.

\section{Material and Methods}

\section{Patient Information}

Sixteen patients diagnosed with brain tumors were considered for the study ( 9 males, 7 females), 
104 aged from 27 to 68 years, with a mean age of $42.62 \pm 10.72$ years (Table 1 ). On the basis of

105 tumor histological features as determined by biopsy and according to the World Health 106 Organization (WHO) criteria ${ }^{35}$, the lesions studied included meningiomas in five patients (Cases $107 \mathrm{M} \# 1-5)$, low-grade gliomas in seven patients (Cases LGG \#1-7), and high-grade gliomas in four 108 patients (Cases HGG \#1-4). The MIB-1 index ${ }^{32}$ was also calculated, and it ranged from 2 to $35 \%$.

109 This study was approved by the local institutional ethics committee on human research of the 110 University Hospital Santa Maria della Misericordia of Udine and clinical data were acquired 111 following the guidelines of the Department of Neurosurgery. Informed consent was obtained 112 from all the patients who participated in this study.

113 Imaging Protocol

114 Diffusion-weighted MRI data were obtained on a 3T scanner (ACHIEVA 3T, Philips, Best, the 115 Netherlands) using a multi-shot DWI-SE sequence (FOV: 240x240x105mm; voxel $1161.0 \times 1.0 \times 1.5 \mathrm{~mm}$; slices acquired parallel to the line connecting the anterior commissure to the 117 posterior commissure, no slice gap; $\mathrm{TE}=74.8 \mathrm{~ms}$; $\mathrm{TR}=8833 \mathrm{~ms}$; $\mathrm{NEX}=1$; parallel reconstruction. 118 Diffusion weightings were isotropically distributed over 64 directions with a b value of 1000 $119 \mathrm{~s}^{*} \mathrm{~mm}^{-2}$, for a total acquisition time of approximately $15 \mathrm{~min}$ ). The MRI sessions also included 120 T1-weighted (3D turbo-gradient-echo sequence, voxel size: 1.000x1.000x1.000 mm; 240 slices) 121 and a Gadolinium-enhanced T1-weighted anatomical MR imaging (3D sagittal-turbo-flash 122 sequence with $\mathrm{TR}=2300 \mathrm{~ms}$, $\mathrm{TE}=2860 \mathrm{~ms}, \mathrm{IR}=1100 \mathrm{~ms}$, flip angle $=20^{\circ}$; voxel size: $1231.000 \times 1.000 \times 1.000 \mathrm{~mm} ; 240$ slices, no gap between slices) was acquired, as well. 
Data analysis was carried out using the Camino software package ${ }^{36}$ (www.camino.org.uk), FSL ${ }^{37}$ (http://www.fmrib.ox.ac.uk/fsl), AFNI $^{38}$ (http://afni.nimh.nih.gov/afni), and ITK-SNAP ${ }^{39}$, as follows.

Preprocessing.

DTI scans were first re-aligned for eddy current-induced geometric distortions and head motion correction using affine registration to the first unweighted (B0) volume ${ }^{40}$. Skull-stripping was then performed using $\mathrm{BET}^{40}$.

Both anatomical T1-weighted scans were rigidly registered to the individual diffusion spaces using the unweighted b0-image to estimate the transformation parameters (Linear Image Registration Tool, FLIRT $\left.^{41}\right)$.

\section{Direction reconstruction.}

A multi-fiber reconstruction approach was followed ${ }^{42}$ and the PAS functions ${ }^{30}$ were computed for each voxel. A reduced encoding approach was adopted ${ }^{43}$, setting the number of basic functions in the PAS representation equal to 16 . The local maxima of the PAS function corresponding to the three principal directions (PDs) were extracted for every voxel using the peak-finding algorithm implemented in the sfpeaks module of the Camino toolkit ${ }^{36}$.

\section{Seed.}

For each patient, a 3D tumoral region of interest (ROI) was manually defined by precisely tracing the contours of the tumor mass slice-by-slice (www.fmrib.ox.ac.uk/fsl/) on the MRI images. Both the unenhanced and enhanced acquired volumes were inspected so that non-tumor 
145 tissue and large vessels were avoided. Subsequently, the ROI was mirrored across the sagittal

146 mid-plane by a procedure included in the ITK-SNAP package ${ }^{39}$ to obtain the homologous region 147 in the healthy hemisphere. A segmentation based on a modified Gaussian mixture model as 148 implemented in $\mathrm{SPM}^{44}$, was performed to produce a whole brain white matter mask. We 149 computed the intersection between the mirrored tumor mask and the white matter one using the 150 voxel-by-voxel arithmetic calculations included in the AFNI software package ${ }^{38}$. The intersection 151 ROI was then transformed from the structural to the native diffusion space with a rigid-body 152 registration, using the $\mathrm{b} 0$ image as a reference ${ }^{40}$. In order to maintain the transformed masks as 153 faithful as possible to those in the native brain images, the masks were conservatively thresholded 154 at 0.9 . Lastly, all the co-registered ROI were visually checked for precision.

155

\section{Tracking - tumor out}

The homologous ROI was used as the "seed" in the tractography analysis (Figure 1: a, b, c). We employed a deterministic streamline tracking algorithm ${ }^{45}$, modified in order to take into account multiple directions per voxe ${ }^{42}$. Streamlines were generated from every point within the seed mask in the healthy hemisphere. The tracking algorithm starts the same number of fibers as the number of PAS peaks in each seed voxel. An anisotropy value less than 0.2 , a curvature of the streamline by more than 60 degrees across a voxel, and entrance of the streamline into an out-ofbrain voxel were used as stopping criteria.

\section{Target Generation .}

Since the main interest was in the long- and medium-range connectivity alterations, an exclusion mask corresponding to a $2 \mathrm{~cm}$ dilation of the tumor region was created ${ }^{39}$ and used to exclude voxels directly neighboring the seed region by means of AFNI voxel-by-voxel arithmetic 
modules $^{38}$. The last ten voxels of each estimated fiber tract were taken into account. The resulting dataset of voxels was visually inspected together with the reconstructed tracks in order to estimate the number of distal clusters to which fibers ran from the tumor-based seed ROI. A kmeans clustering algorithm developed in-house was applied to identify the specific centroids of these clusters. When fibers projected to two diametrically opposed brain areas, two centroids, one for each area, the ones pointed by most of the tracked pathways, were taken into account. In cases where fibers projected from the seed to nearby brain areas, only the centroid pointed by most of the projections was considered. This in order to differentiate if the contralateral region acted as an interconnecting node between two distal brain area or as a seed station. Subsequently, since there are asymmetric tracts in the human brain ${ }^{46}$, a wide ROI (10mm-diameter sphere) around each defined centroid of interest was drawn ${ }^{39}$ in order to ensure the reconstruction of all likely fiber tracts in both hemispheres. By this way, target regions were obtained having a relevant connectivity with the tumor volume mirrored in the non-affected side.

\section{Tracts of interest.}

The lesion-based seeds and target ROIs, on both the affected and contralateral sides, were used in several ways to provide a complete evaluation of the impact of the tumor on WM fiber architecture.

Another tracking framework, based on a two ROI analysis ${ }^{47}$, was used to infer connectivity between the delineated areas of interest in both the affected and the contralateral hemispheres. We ran deterministic tractography to generate streamlines from every point within the brain mask, this time used as the "seed". Bending angle and fractional anisotropy criteria for this second tractography analysis were the same in both procedures (60 degree, 0.2 ). After that, where diametrically opposed target areas were seen relative to the tumor (Cases M3, M4, LGG1, and 
LGG5), the WM bundles connecting them were investigated to estimate the fiber tracts which would have presumably run through the tumor. The two selected target ROIs were so applied as endpoint masks to prune the tracked bundles and to reconstruct streamlines connecting them in the healthy hemisphere. Finally, the target regions were sagittally mirrored and the tracts of interest in the affected hemisphere generated in the same manner as described above for the healthy hemisphere (Figure 1: d1, e1).

In cases where only one target region was considered (Cases M1, M2, M5, LGG2, LGG3, LGG4, LGG6, LGG7, and HGG \#1-4), to make inferences about possible distortion of the WM in the neighborhood of the tumor, the tumor-based ROIs were dilated using SPM morphology operators to 1.2 times their original size. This expanded ROI and the target area were then used as endpoints in the two ROI analysis of WM tract density. Subsequently, both the target and the dilated homologous ROI were sagittally flipped, and the correspondent tracts were mapped in the pathological hemisphere through the corresponding tracking process (Figure 1: d2, e2).

Based on the results of this tractography procedure, two comparisons were undertaken: Comparison between hemispheres and Comparison against probabilistic tractography, as described below.

\section{Comparison between hemispheres.}

A numerical quantification of tumor-induced WM changes was assessed by counting the estimated fiber tracts of interest in each hemisphere. The percentage of decrease in tract fiber count on the pathological side was computed for every patient, taking as reference the tracts of the healthy hemisphere. Changes were assessed in relation to MIB1 index using a simple linear regression in order to investigate the relationship between severity of tumor-induced damages and lesion-specific histological features. In particular, for each case, we weighted the percentage 
213 of tracts decrease by dividing tracts value by individual patient's estimated tumor size in order to

214 account for the relation between amount of disruption and extension of the lesion. The tumor

215 volume was computed from the lesion mask previously mentioned, taking into account the voxel

216 size (Table 1).

217 Finally, for each patient, the reconstructed trajectories were rendered on the 3D brain and tumor 218 ROI to better display the spatial relationship between WM tracts and lesion.

219 Comparison against probabilistic tractography

220 To verify whether the deterministic PAS-MRI process accurately determines all the WM 221 connections and to obtain a measure about the confidence of the reconstructed trajectories, the 222 pathways under examination in the two hemispheres were also defined in a probabilistic 223 framework. In particular, we prepared a parametric model of uncertainty, computing a probability 224 density function (PDF) of the diffusion orientations in each voxel for the multi-fiber population 225 cases, according to the PAS reconstruction approach. Tractography was achieved by iterative 226 streamline propagation through the computed PDFs for each PD estimated, using 1000 iterations.

227 The same tracking procedure of the deterministic framework was adopted: seeding everywhere in 228 the brain volume and subsequently identifying streamlines connecting the ROIs. For each subject, 229 the resulting collection of fiber tracks (i.e., across tumor -or mirrored tumor- and target ROIs) 230 was combined to form a patient-specific connection probability image, where each voxel contains 231 the number of streamlines that enter the voxel divided by the total number of streamlines in the 232 patient's collection of fiber tracts. Within patients, probabilistic and deterministic reconstructed 233 tract volumes were visually compared in each hemisphere as well as between hemispheres, 234 overlaying the two outputs on the same slices. For probabilistic tractography, voxels with the 235 highest connectivity were considered those most likely to be part of the connecting bundles of 
237 Results

238 Mapping the WM bundles of interest in the pathological hemisphere, after assessing them 239 in the unaffected one, revealed different types of WM involvement by brain tumors which 240 strongly correlated with the histopathological features of the lesions. Table 1 summarizes the 241 alterations of fiber tracts, as well as some clinical and anatomical data concerning the patients. 242 The higher the MIB-1 label was, the more fibers were found to have been destroyed, with a 243 correlation coefficient equal to $0.83(\mathrm{p}=0.0001)$, as shown in Figure 2.

244 Meningiomas

In patients M1 and M2, the fiber pathway depicted in the healthy hemisphere were found to 246 be splayed into different branches in the affected hemisphere as a result of the tumor mass 247 (Figure 3). Nevertheless, the tractography output showed a peripheral region of intact white matter at the tumor boundary where the fiber tracts were still identifiable. Indeed, in the 249 pathological hemisphere, the streamline algorithm did not find under-threshold anisotropy areas, 250 and it reconstructed the connection pathway under examination. This was confirmed by 251 comparing the counts of the estimated fiber tracts in both hemispheres. Most (over two-thirds) of 252 the connecting streamlines identified in the healthy hemisphere were tracked also in the lesioned 253 hemisphere (Table 1).

254 In the other three meningioma cases, deterministic tractography clearly suggested bulk 255 displacement of WM tracts associated with tumor (Figure 3). The lesion mass changed the 256 location and organization of WM pathways but only slightly affected the coherence of fiber 
bundles. Indeed, in all cases of meningioma, we observed a modest decrease in the number of estimated fiber tracts in the lesioned hemisphere $(10-32 \%)$, as compared to the healthy WM architecture (Table 1). The average ( \pm standard deviation $[\mathrm{SD}]$ ) percent difference in the number of estimated tracts between the two hemispheres across the 5 cases of meningioma was $17.75 \pm$ $9.16 \%$. This indicates that the underlying axonal structures have remained intact but spatially displaced.

\section{Gliomas}

Low-Grade: In patient LGG1, two main connecting fiber bundles were tracked in the healthy WM architecture. The superior bundle of Cingulum, reconstructed in the homologous region resulted as being dorsally displaced in the pathological hemisphere. Moreover, the inferior pathway was not identified at all in the lesioned area, probably as a result of tumor-induced destruction. Case LGG5 showed clear displacement of the Inferior Fronto-Occipital Fasciculus under consideration, though the tumor mass destroyed a large part of the fiber tracts. Two main connecting bundles of the Arcuate Fasciculus were also seen for patient LGG3. Here however, the inferior pathway did not have a correspondent on the affected side. The number of fibers in the other main bundle identified in the healthy hemisphere was notably reduced in the proximity of the tumor. In the other low-grade gliomas (Cases LGG2, LGG4, LGG6, and LGG7), the tractography showed that glioma did not change the location of the connecting pathway, but lead to a reduction in the number of tracked fibers (Figure 4).

The number of estimated fiber tracts in the connection pathways studied was noticeably reduced on the pathological side as compared to contralateral WM architecture (25-90\%, Table 1). Across these seven cases of low-grade glioma, the average $( \pm \mathrm{SD})$ percentage of tract reduction due to tumor infiltration was $61.9 \pm 28.05 \%$, indicating a high variability in their malignancy. 
High-Grade: Evidence of white matter tract infiltration was presented by all four patients with high-grade glioma (HGG \#1-4). The fiber bundles crossing the tumor in the affected hemisphere were extremely reduced, as compared to the contralateral hemisphere ( $90-96 \%$, Table 1). Tractography results clearly demonstrated this loss of fibers with no displacement of white matter architecture, which is suggestive of tumor invasion (Figure 5). Across the four high-grade gliomas, an average $( \pm \mathrm{SD})$ percent decrease in tract fiber counts equal to $93.53 \pm 2.78 \%$ was found, with respect to non-affected hemisphere.

The difference in percentage of lesion-induced decrease in tracts between the two main groups (meningiomas and gliomas) was statistically significant $(\mathrm{p}=0.0006)$.

The connection pathways as assessed by using probabilistic tractography confirmed the WM alteration patterns identified in each patient. The connectivity architecture obtained with the probabilistic approach corresponded well with that showed by our technique by deterministic tracking. In every patient the two types of connection analyses substantially coincided and no outlier from deterministic tracking were found, indicating that the deterministic choice did not compromise the quality of the results and captured all the connecting bundles. Three exemplificative cases of this comparisons between the two approaches are reported in Figure 6.

\section{Discussion}

In this study, we present a novel approach for defining alterations in WM paths induced by brain lesions by using diffusion tractography. Our method does not take into account anatomically predefined fiber tracts, and hence no a priori knowledge of the pathways under investigation is 
required.

In the proposed approach, the adoption of PAS-MRI as direction reconstruction method for multifiber deterministic tractography, reduced the classic limitation of the diffusion tensor model and its inability to differentiate tracts in cases of WM fiber crossing, branching or fanning. Our choice overcame the underestimation of the extent of tracks of interest that may bring to unreliable and clinically misleading information ${ }^{48}$, although in a clinically compatible acquisition time.

The results for meningioma show displacements of the WM pathways, indicating the presence of intact but spatially deviated axonal structures. Meningioma patients maintained a degree of connectivity sufficiently comparable to the contralateral hemisphere. Indeed, brain tumors like meningiomas do not usually infiltrate the surrounding brain tissue, but simply compress or distort $\mathrm{it}^{49,50}$. In patients with low-grade glioma, a mixed pattern of tract deviation and disruption was seen, again, consistent with previous studies ${ }^{51,52}$. All the analyzed cases share a common feature. Low-grade gliomas are usually characterized by an extensive, diffuse infiltration of tumoral cells that preferentially invade the brain along myelinated fibers in white matter $\operatorname{tracts}{ }^{53}$. The mass effect of the lesion bulk appeared to be insufficient to account for this reduction in fiber tracts, which should most likely be considered as an index of WM disruption caused by edema/tumor infiltration. In the presence of high-grade glioma, tractography analysis showed an almost complete disruption of the fiber bundles ${ }^{54,55}$.

Our method used the percentage of decrease in tracts as a measure of the severity of WM alterations caused by the tumor mass. This data correlated with histopathological tumoral features. Indeed, our data presented significantly different degrees of WM involvement in the two groups of patients (glioma and meningioma). In addition, a strong correlation was found between the amount of fibers disrupted and the MIB-1 index.

In the proposed technique, we adopted deterministic tractography for both speed and 
simplicity of use. The technique could however, be adapted to use probabilistic tracking, which is an optimal method for modeling uncertainty, generating multiple curves from a seed point. This method ensures a greater robustness to the image noise ${ }^{32}$.

As part of our evaluation, we compared the results of our technique with those obtained using a probabilistic framework. The latter step of the analysis was so time-consuming (requiring several days for each case) that it could not be applied in a preoperative routine setting, though this may change with hardware and algorithmic improvements. In our specific cohort, the results of probabilistic tractography showed our deterministic results obtained with PAS-MRI to yield substantially equivalent results. The consistency of our results with those of probabilistic tractography supports the assessment of differences in various types of tumors by observing WM pathway changes and suggests the feasibility of the proposed method in those cases in which the lesion does not involve major/known WM pathways and a priori information about the local fibers anatomy is lacking.

\section{Limitations of the Study}

We are aware that our work is based on a relatively small sample size. Nonetheless, the results obtained in this preliminary study and their congruence in the various patients belonging to the three main classes of tumors, suggest stereotypical tumor-induced WM alterations, in relation to the type and the severity of the lesion.

The inhomogeneity of tumor locations and sizes creates a difficulty in that some tracts may be intersected by the tumor in mid-tract, whilst others effectively extend in only one direction from the tumor area. To accommodate these two contexts, we incorporated a dual ROI tracking approach between target ROIs when apparent tract passage was seen in the original tracking from the homologous tumor ROI, and used the target ROI and the tumor (or tumor homolog) ROI for 
tracking otherwise. The choice as to which approach to apply, however, was made on a subjective basis and a set of objective criteria for determining which one would be desirable to perform in a given case. It is unlikely that this aspect of our technique influenced the comparisons performed as the methods were applied bilaterally for comparison at the patient level, and were mixed across the patient groups.

In the present work, we didn't take into account pre- or peri-operative functional localization findings. When such information is available, either from fMRI or from intraoperative cortical/subcortical electrodes, the computation speed of the deterministic approach (or the recalculation of tract probability distributions) could facilitate the incorporation of such data into surgical guidance systems ${ }^{55}$.

We used five software packages to analyze our data in order to take advantage of the specialized algorithms specific of the various packages. In view of potential use in clinical routine, a uniform interface that combines all the processing steps within a single framework would be useful.

Multi-shot echo-planar imaging (EPI) was adopted. This achieve higher spatial resolution to fully capture the complex axonal configurations and limit the partial volume effects enhanced from the anisotropic-voxel acquisition performed, at the expense of more susceptiblity to motion. HARD tractography methods, such as the one we used, greatly improved resolution for detecting crossing fibers, but are still susceptible to errors introduced by uncertainty at sites of fiber crossing. This is typical of any deterministic tractography analysis especially with T2weighted signal abnormalities in the proximity or within the lesion. Comparison with a complementary probabilistic analysis showed that this was not a significant problem in our cohort of patients. 
371

372 Diffusion imaging has become an essential part of MRI examinations in brain tumor patients. To 373

374

375

376

377

378

379

380

381

382

383

384 The authors declare no competing financial interests. 
1. Field AS, Alexander AL, Wu YC, Hasan KM, Witwer B, Badie B. Diffusion Tensor

Eigenvector Directional Color Imaging Patterns in the Evaluation of Cerebral White Matter

Tracts Altered by Tumor. J Magn Reson Imaging 2004; 20: 555-562.

2. Jellison BJ, Field AS, Medow J, Lazar M, Salamat MS, Alexan-der AL. Diffusion Tensor Imaging of Cerebral White Matter:A Pictorial Review of Physics, Fiber Tract Anatomy, and Tumor Imaging Patterns. AJNR Am J Neuroradiol 2004; 25: 356-369.

3. Skrap M, Mondani M, Tomasino B, Weis L, Budai R, Pauletto G, Eleopra R, Fadiga L, Ius T. Surgery of insular nonenhancing gliomas: volumetric analysis of tumoral resection, clinical outcome, and survival in a consecutive series of 66 cases. Neurosurgery 2004; 70(5), 1081-1094.

4. Clark CA, Barrick TR, Murphy MM, Bell BA. 2003. White matter fiber tracking in patients with space-occupying lesions of the brain:a new technique for neurosurgical planning? Neuroimage 2003; 20: 1601-1608.

5. Basser PJ, Mattiello J, Le Bihan D. MR diffusion tensor spectroscopy and imaging. Biophys J $1994 ; 66: 259-267$.

6. Conturo TE, Lori NF, Cull TS, Akbudak E, Snyder AZ, Shimony JS, McKinstry RC, Burton H,

Raichle ME. Tracking neuronal fiber pathways in the living human brain. Proc. Natl. Acad. Sci 1999; USAv96: 10422 - 10427.

7. Mori S, Crain BJ, Chacko VP, van Zijl PCM. Three dimensional tracking of axonal projections in the brain by magnetic resonance imaging. Annals of Neurology 1999; 45:265-269.

8. Basser PJ, Pajevic S, Pierpaoli C, Duda J, and Aldroubi A. In vivo fiber tractography using DTMRI data. Magnetic Resonance in Medicine 2000; 44:625-632. 
408

409

410

411

412

413

414

415

416

417

418

419

420

421

422

423

424

425

426

427

428

429

430

L. Predictive value of inferior fronto-occipital fasciculus (IFO) DTI-fiber tracking for determining the extent of resection for surgery of frontal and temporal gliomas preoperatively. $\mathrm{J}$ Neurosurg Sci. :2012; 56(2):137-43.

10. Zolal A, Hejčl A, Vachata P, Bartoš R, Humhej I, Malucelli A, Nováková M, Hrach K, Derner

M, Sameš M. The use of diffusion tensor images of the corticospinal tract in intrinsic brain tumor surgery: a comparison with direct subcortical stimulation. Neurosurgery 2012; 71(2):331-40.

11 Lazar M, Alexander AL, Thottakara PJ, et al. White matter reorganization after surgical resection of brain tumors and vascular malformations, AJNR Am J Neuroradiol 2006; 27:125871.

12. Xu JL, Li YL, Lian JM, Dou SW, Yan FS, Wu H, Shi DP. Distinction between postoperative recurrent glioma and radiation injury using MR diffusion tensor imaging. Neuroradiology 2010; 52(12):1193-9.

13. Kovanlikaya I, Firat Z, Kovanlikaya A, Uluğ AM, Cihangiroglu MM, John M, Bingol CA, Ture U. Assessment of the corticospinal tract alterations before and after resection of brainstem lesions using Diffusion Tensor Imaging (DTI) and tractography at 3T. Eur J Radiol. 2011; 77(3): 383-91.

14. Nowosielski M, Recheis W, Goebel G, Güler O and Tinkhauser G, et al. ADC histograms predict response to anti-angiogenic therapy in patients with recurrent high-grade glioma. Neuroradiology 2011; 53(4): 291-302.

15. Kinoshita M, Hashimoto N, Gogo T, et al. Fractional anisotropy and tumor cell density of the tumor core show positive correlation in diffusion tensor magnetic resonance imaging of malignant brain tumors, NeuroImage 2008; 43:29-35.

16. Byrnes TJ, Barrick TR, Bell BA, Clark CA. Diffusion tensor imaging discriminates between 
glioblastoma and cerebral metastases in vivo. NMR Biomed. 2011; 24(1):54-60.

17. Chen Y, Shi Y, Song Z. Differences in the architecture of low-grade and high-grade gliomas evaluated using fiber density index and fractional anisotropy. J Clin Neurosci 2010; 17(7):824-9.

18. Jolapara M, Kesavadas C, Radhakrishnan VV, Thomas B, Gupta AK, Bodhey N, Patro S, Saini J, George U, Sarma PS. Role of diffusion tensor imaging in differentiating subtypes of meningiomas. J Neuroradiol. 2010; 37(5):277-83.

19. Clark CA, Barrick TR, Murphy MM, Bell BA. White matter tracking in patients with spaceoccupying lesions of the brain: a new technique for neurosurgical planning? NeuroImage 2003; 20: $1601-1608$.

20. Yamada K, Kizu O, Mori S, Ito H, Nakamura H, Yuen S, Kubota T.Tanaka O, Akada W, Sasajima H, Mineura K, Nishimura T. Brain fiber tracking with clinically feasible diffusion tensor MR imaging: Initial experience. Radiology 2003; 227:295 - 301.

21. Morita N, Wang S, Kadakia P, Chawla S, Poptani H, Melhem ER. Diffusion tensor imaging of the corticospinal tract in patients with brain neoplasms. Magn Reson Med Sci. 2011; 10(4):23943.

22. Bobek-Billewicz B, Stasik-Pres G, Majchrzak K, Senczenko W, Majchrzak H, Jurkowski M, Połetek J. Fibre integrity and diffusivity of the pyramidal tract and motor cortex within and adjacent to brain tumour in patients with or without neurological deficits. Folia Neuropathol. $2011 ; 49(4): 262-70$.

23. Pantelis E, Papadakis N, Verigos K, Stathochristopoulou I, Antypas C, Lekas L, Tzouras A, Georgiou E, Salvaras N. 2010. Integration of functional MRI and white matter tractography in stereotactic radiosurgery clinical practice. Int J Radiat Oncol Biol Phys. 2010; 78(1):257-67. 
454

455

456

457

458

459

460

461

462

463

464

DTI fiber tracking techniques on their clinical applications in patients with brain tumors. Neuroradiology 2010; 52(1):37-46.

25. Bucci M, Mandelli ML, Berman JI, Amirbekian B, Nguyen C, Berger MS, Henry RG. Quantifying diffusion MRI tractography of the corticospinal tract in brain tumors with deterministic and probabilistic methods. Neuroimage Clin. 2013 Aug 20;3:361-8.

26. Giussani C, Roux FE, Ojemann J, Sganzerla EP, Pirillo D, Papagno C. Is preoperative functional magnetic resonance imaging reliable for language areas mapping in brain tumor surgery? Review of language functional magnetic resonance imaging and direct cortical stimulation correlation studies. Neurosurgery. 2010 Jan;66(1):113-20. doi: 10.1227/01.NEU.0000360392.15450.C9.

27. Peled S, Friman O, Jolesz F, Westin CF. Geometrically constrained two-tensor model for crossing tracts in DWI. Magn Reson Imaging. 2006 Nov;24(9):1263-70. Epub 2006 Sep 12.

28. Tournier JD, Calamante F, Connelly A. Robust determination of the fibre orientation distribution in diffusion MRI: non-negativity constrained super-resolved spherical deconvolution. Neuroimage. 2007 May 1;35(4):1459-72. Epub 2007 Feb 21.

29. Sotiropoulos SN, Bai L, Morgan PS, Auer DP, Constantinescu CS, Tench CR. A regularized two-tensor model fit to low angular resolution diffusion images using basis directions. J Magn Reson Imaging. 2008 Jul;28(1):199-209.

30. Jansons K, Alexander D. Persistent angular structure: new insights from diffusion magnetic resonance imaging data. Inverse Probl. 2003; 19(5):1031 - 1046.

31. Bertani G, Carrabba G, Raneri F, Fava E, Castellano A, Falini A, Casarotti A, Gaini SM, Bello L. Predictive value of inferior fronto-occipital fasciculus (IFO) DTI-fiber tracking for determining the extent of resection for surgery of frontal and temporal gliomas preoperatively. $\mathrm{J}$ Neurosurg Sci. :2012; 56(2):137-43. 
32. Behrens TE, Woolrich MW, Jenkinson M, Johansen-Berg H, Nunes RG, Clare S, Matthews PM, Brady JM, Smith SM. Characterization and propagation of uncertainty in diffusion-weighted MR imaging. Magn Reson Med. 2003 Nov;50(5):1077-88.

33. Parker GJM, Haroon HA, Wheeler-Kingshott CAM. A framework for a streamline-based probabilistic index of connectivity (PICo) using a structural interpretation of MRI diffusion measurements. J Magn Reson Imaging 2003;18:242-54.

34. Brat DJ, Prayson RA, Ryken TC, Olson J. Diagnosis of malignant glioma: role of neuropathology, J Neurooncol 2008; 89:287-311.

35. Louis DN, Ohgaki H, Wiestler OD, Cavenee WK, Burger PC, Jouvet A, Scheithauer BW, Kleihues P. The 2007 WHO Classification of Tumours of the Central Nervous System Acta Neuropathol 2007 Aug;114(2):97-109. Epub 2007 Jul 6.

36. Cook PA, Bai Y, Nedjati-Gilani S, Seunarine KK, Hall MG, Parker GJ, Alexander DC, Camino: Open-Source Diffusion-MRI Reconstruction and Processing, 14th Scientific Meeting of the International Society for Magnetic Resonance in Medicin, Seattle, WA, USA, p. 2759, May 2006.

37. Smith SM, Jenkinson M, Woolrich MW, Beckmann CF, Behrens TEJ, Johansen-Berg H, Bannister PR, De Luca M, Drobnjak I, Flitney DE, Niazy R, Saunders J, Vickers J, Zhang Y, De Stefano N, Brady JM, Matthews PM, Advances in functional and structural MR image analysis and implementation as FSL. NeuroImage 2004; 23(S1):208-219.

38. Cox RW. AFNI: software for analysis and visualization of functional magnetic resonance neuroimages. Comput Biomed Res. 1996; 600: 29:162-173.

39. Yushkevich PA, Piven J, Hazlett HC, Smith RG, Ho S, Gee JC, Gerig G. User-guided 3D active contour segmentation of anatomical structures: Significantly improved efficiency and reliability. Neuroimage 2006; 1; 31(3):1116-28. 
502

503

504

505

506

507

508

509

510

511

512

513

514

40. Smith SM. Fast robust automated brain extraction, Hum Brain Mapp 2002; 17:143-155.

41. Jenkinson M, Smith SM. A global optimisation method for robust affine registration of brain images. Medical Image Analysis, 5(2):143-156, 2001.

42. Seunarine K, Cook P, Hall M, Embleton K, Parker G, Alexander D. Exploiting peak anisotropy for tracking through complex structures. IEEE ICCV Workshop on MMBIA, 2007.

43. Sweet and Alexander, Reduced Encoding Persistent Angular Structure, 572 ISMRM, 2010.

44. Ashburner J, Friston KJ. Unified segmentation. Neuroimage 2005; 26:839-851.

45. Mori S, Crain BJ, Chacko VP, van Zijl PCM. Three-dimensional tracking of axonal projections in the brain by magnetic resonance imaging, Annals of Neurology 1999; 45:265-269.

46. Thiebaut de Schotten M, Ffytche DH, Bizzi A, Dell'Acqua F, Allin M, Walshe M, Murray R, Williams SC, Murphy DG, Catani M. Atlasing location, asymmetry and inter-subject variability of white matter tracts in the human brain with MR diffusion tractography. Neuroimage. 2011 Jan $1 ; 54(1): 49-59$.

47. Campanella M, Molinari E, Baraldi P, Nocetti L, Porro CA, Alexander DC. An algorithm to estimate anatomical connectivity between brain regions using diffusion MRI, MRI 2012: Volume 31 , Issue 32 .

48. Farquharson S, Tournier JD, Calamante F, Fabinyi G, Schneider-Kolsky M, Jackson GD, Connelly. White matter fiber tractography: why we need to move beyond DTI. A.J Neurosurg. 2013 Jun;118(6):1367-77. doi: 10.3171/2013.2.JNS121294. Epub 2013 Mar 29.

49. Corie W. Wei, Gang Guo, David J. MikulisTumor Effects on Cerebral White Matter as Characterized by Diffusion TensorTractography, Can. J. Neurol. Sci. 2007; 34: 62-69

50. Carvi y Nievas MN, Hoellerhage HG, Drahten C. White matter tract alterations assessed with diffusion tensor imaging and tractography in patients with solid posterior fossa tumors. Neurol 
India 2010;58:914-21

51. Kuhnt D, Bauer MH, Egger J, Richter M, Kapur T, Sommer J, Merhof D, Nimsky CFiber tractography based on diffusion tensor imaging compared with high-angular-resolution diffusion imaging with compressed sensing: initial experience. Neurosurgery. 2013 Jan;72 Suppl 1:165-75. doi: 10.1227/NEU.0b013e318270d9fb.

52. Castellano A, Bello L, Michelozzi C, Gallucci M, Fava E, Iadanza A, Riva M, Casaceli G, Falini A, Role of diffusion tensor magnetic resonance tractography in predicting the extent of resection in glioma surgery. Neuro Oncol. 2012 Feb;14(2):192-202. doi: 10.1093/neuonc/nor188. Epub 2011 Oct 20

53. Duffau H, Khalil I, Gatignol P, Denvil D, Capelle L. Surgical removal of corpus callosum infiltrated by low-grade glioma: functional outcome and oncological considerations. J Neurosurg 2004;100(3):431-7.

54. Gulati S, Berntsen EM, Solheim O, Kvistad KA, Håberg A, Selbekk T, Torp SH, Unsgaard G.Minim. Surgical resection of high-grade gliomas in eloquent regions guided by blood oxygenation level dependent functional magnetic resonance imaging, diffusion tensor tractography, and intraoperative navigated 3D ultrasound. Invasive Neurosurg. 2009 Feb; 52(1):17-24. doi: 10.1055/s-0028-1104566. Epub 2009 Feb 26

55. Chen F, Zhang X, Li M, Wang R, Wang HT, Zhu F, Lu DJ, Zhao H, Li JW, Xu Y, Zhu B, Zhang B, Axial diffusivity and tensor shape as early markers to assess cerebral white matter damage caused by brain tumors using quantitative diffusion tensor tractography. CNS Neurosci Ther. 2012 Aug;18(8):667-73. doi: 10.1111/j.1755-5949.2012.00354.x. Epub 2012 Jun 19. 


\section{Table 1 (on next page)}

Clinical information and outcomes in the cohort of patients. 
MENINGIOMA

\begin{tabular}{|c|c|c|c|c|c|c|}
\hline Case No. & Age(yrs) & Sex & Tumor Location & Tumor Size [cm3] & MIB- & Percentage Decrease in Tract \\
\hline & & & & & $1 \%$ & Count \\
\hline M1 & 68 & $\mathrm{M}$ & RH Premotor Motor & 11.67 & 2 & $10.41 \%$ \\
\hline M2 & 55 & M & LH Parietal Motor & 52.99 & 2 & $21.25 \%$ \\
\hline M3 & 48 & $\mathrm{~F}$ & LH Temporal Parietal Motor & 36.16 & 4 & $10.00 \%$ \\
\hline M4 & 27 & $\mathrm{~F}$ & RH Temporal Motor Premotor & 106.25 & 4 & $32.00 \%$ \\
\hline M5 & 35 & M & LH Premotor Motor & 43.03 & 10 & $15.10 \%$ \\
\hline \multicolumn{7}{|c|}{ LOW GRADE GLIOMA } \\
\hline LGG1 & 28 & $\mathrm{~F}$ & LH Insular Temporal & 107.4 & 3 & $45.10 \%$ \\
\hline LGG2 & 37 & $\mathrm{~F}$ & RH Frontal & 13.29 & 4 & $24.68 \%$ \\
\hline LGG3 & 37 & $\mathrm{~F}$ & LH Frontal Premotor & 53.38 & 4 & $28.72 \%$ \\
\hline LGG4 & 38 & M & LH Frontal Premotor & 98.83 & 4 & $81.25 \%$ \\
\hline LGG5 & 46 & M & LH Temporal Occipital Insular & 43.2 & 9 & $81.81 \%$ \\
\hline LGG6 & 50 & M & RH Insular Temporal Parietal & 46.15 & 9 & $81.85 \%$ \\
\hline LGG7 & 40 & M & RH Premotor & 11.16 & 35 & $89.92 \%$ \\
\hline \multicolumn{7}{|c|}{ HIGH GRADE GLIOMA } \\
\hline HGG1 & 41 & $\mathrm{~F}$ & RH Temporal Motor Parietal & 45.53 & 12 & $95.58 \%$ \\
\hline HGG2 & 51 & $\mathrm{~F}$ & RH Frontal PremotorMotor & 46.62 & 18 & $96.18 \%$ \\
\hline HGG3 & 32 & M & LH Frontal Premotor & 21.8 & 25 & $90.43 \%$ \\
\hline HGG4 & 49 & M & LH Premotor Motor Insular & 43.02 & 30 & $91.95 \%$ \\
\hline
\end{tabular}




\section{Figure 1}

Figure 1: Method schematics

(a) 3D tumor ROI was identified from the anatomical MRI image.

(b) The ROI was mirrored across the sagittal mid-plane to obtain the homologous region in the healthy hemisphere. A segmentation was performed and the intersection between the flipped tumor ROI and the white matter mask was computed; (c) The intersection mask was then transformed from the structural to the native diffusion space and used as the "seed" in the deterministic tractography analysis. Streamlines were generated from every point within the seed mask in the healthy hemisphere and two (d1) or only one (d2) target regions were identified; (e1) In the first case the WM bundles connecting the two target ROls were investigated. The targets were sagittally mirrored and, after seeding, the tracts of interest in the lesioned hemisphere were generated in the same manner. (e2) In the second case the homologous region mask was dilated and the streamlines connecting the dilated $\mathrm{ROI}$ and the target were tracked to estimate the contralateral WM tracts involved in the tumor area.

Subsequently, both the target and the dilated homologous $\mathrm{ROI}$ were sagittally flipped and the contralateral tracts were reconstructed in the pathological hemisphere; (e1)-(e2) The tracts of interest in the two hemispheres were finally defined in a probabilistic framework following the same tracking procedures and according to the number of targets identified. Single connection probability images were thus obtained to confirm deterministic tracking results. 
Definition of the Seed

a)

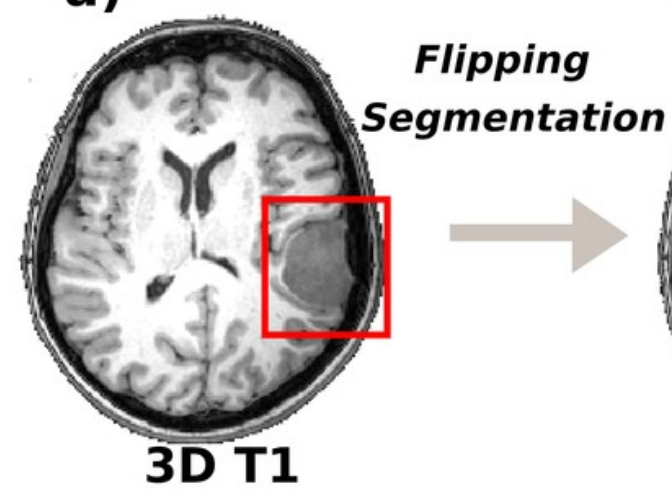

b)

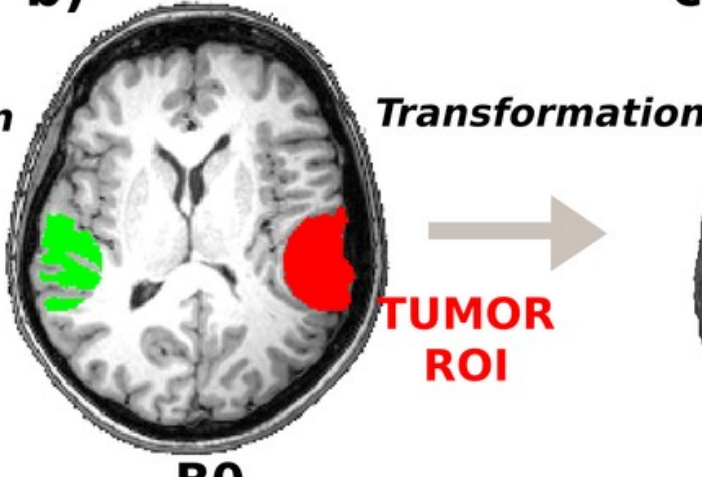

c)
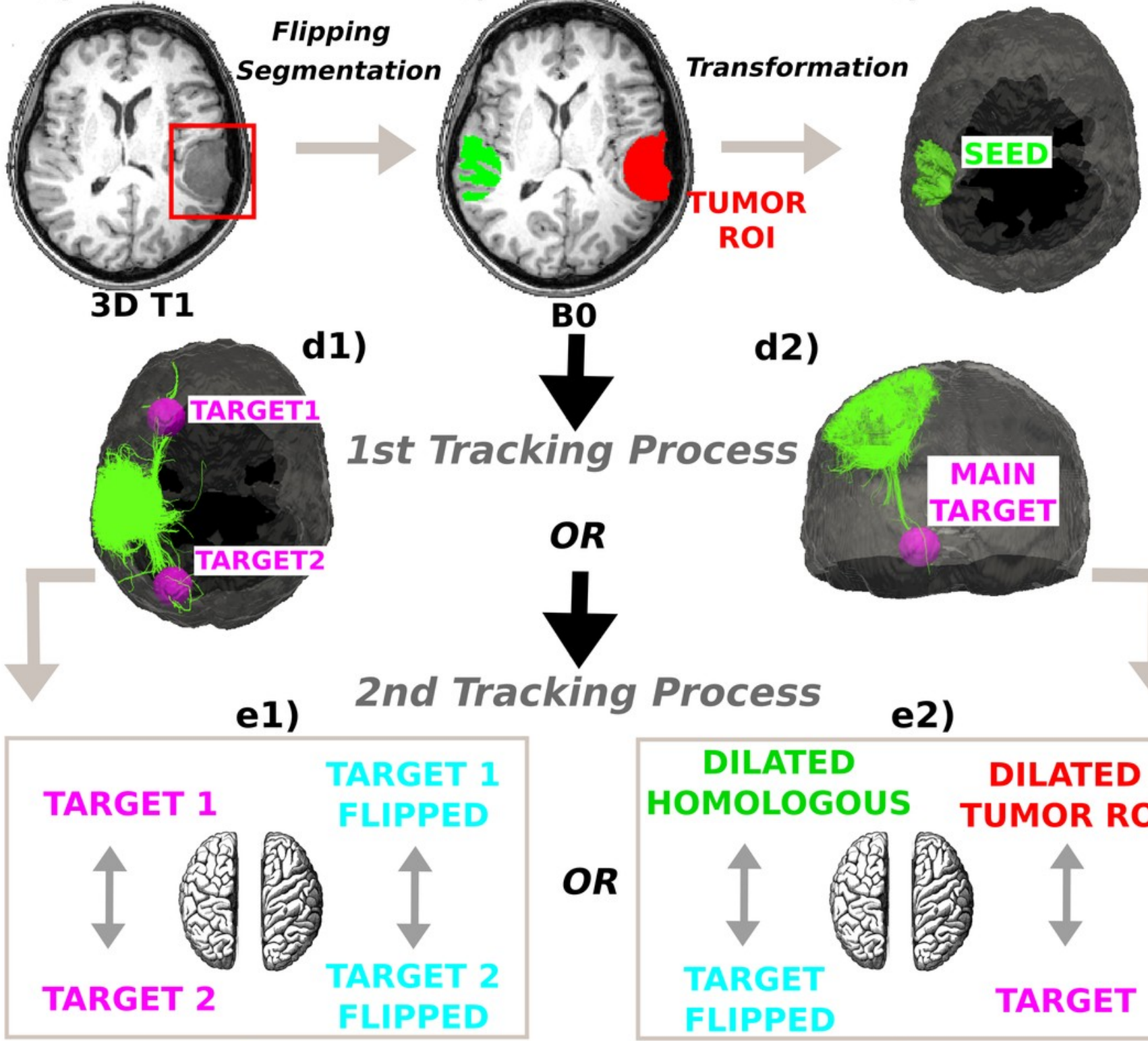

d2)

OR
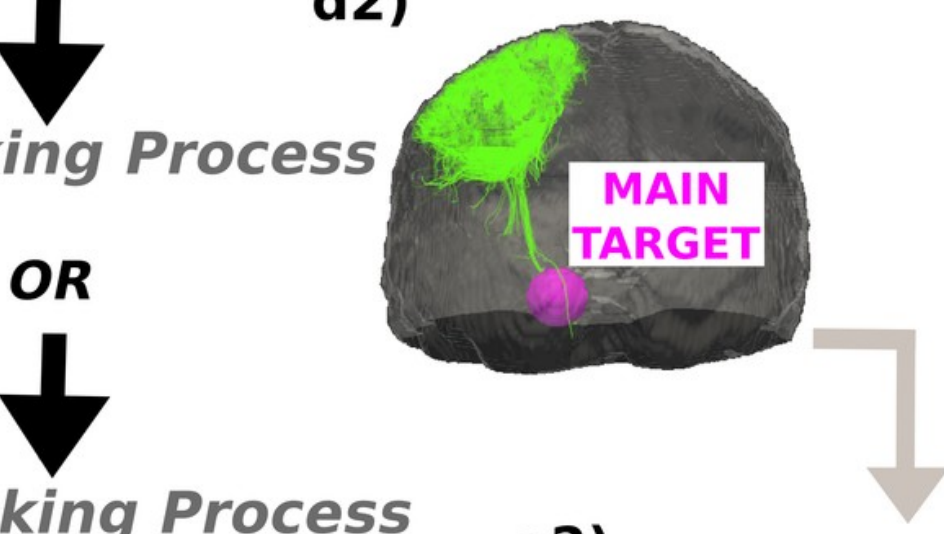

e2)

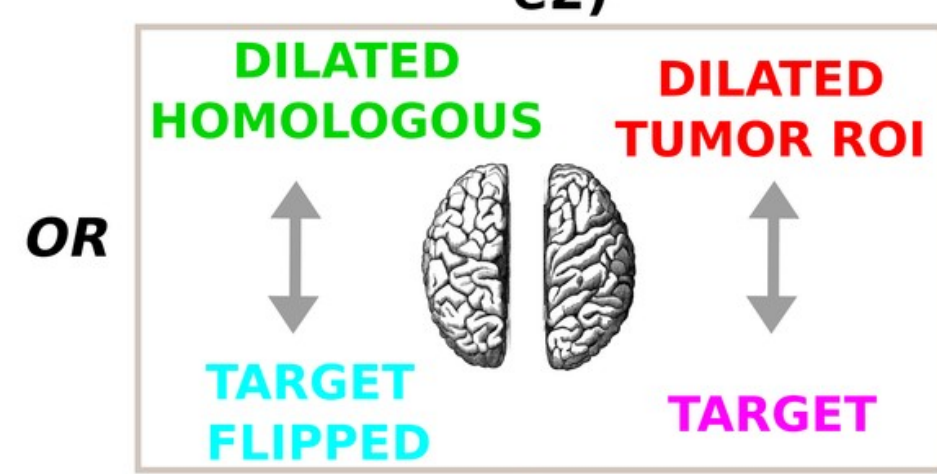

f1)

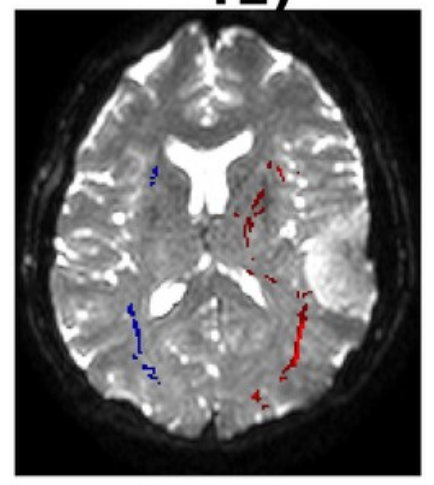

Probabilistic Analysis f2)

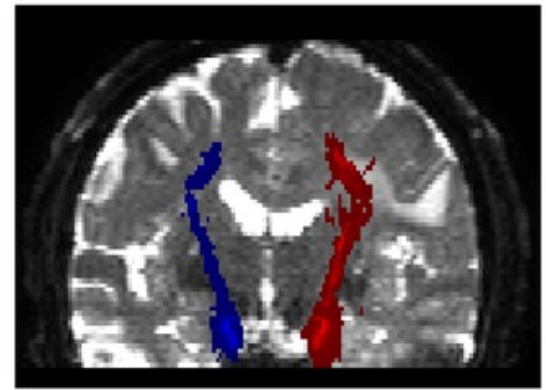




\section{Figure 2}

Figure 2

Linear regression between the pondered tumor-induced reductions in tracts and lesionspecific histological features (MIB-1 index) across all the studied cases; scatter plot shows the data and the estimated linear fitting.

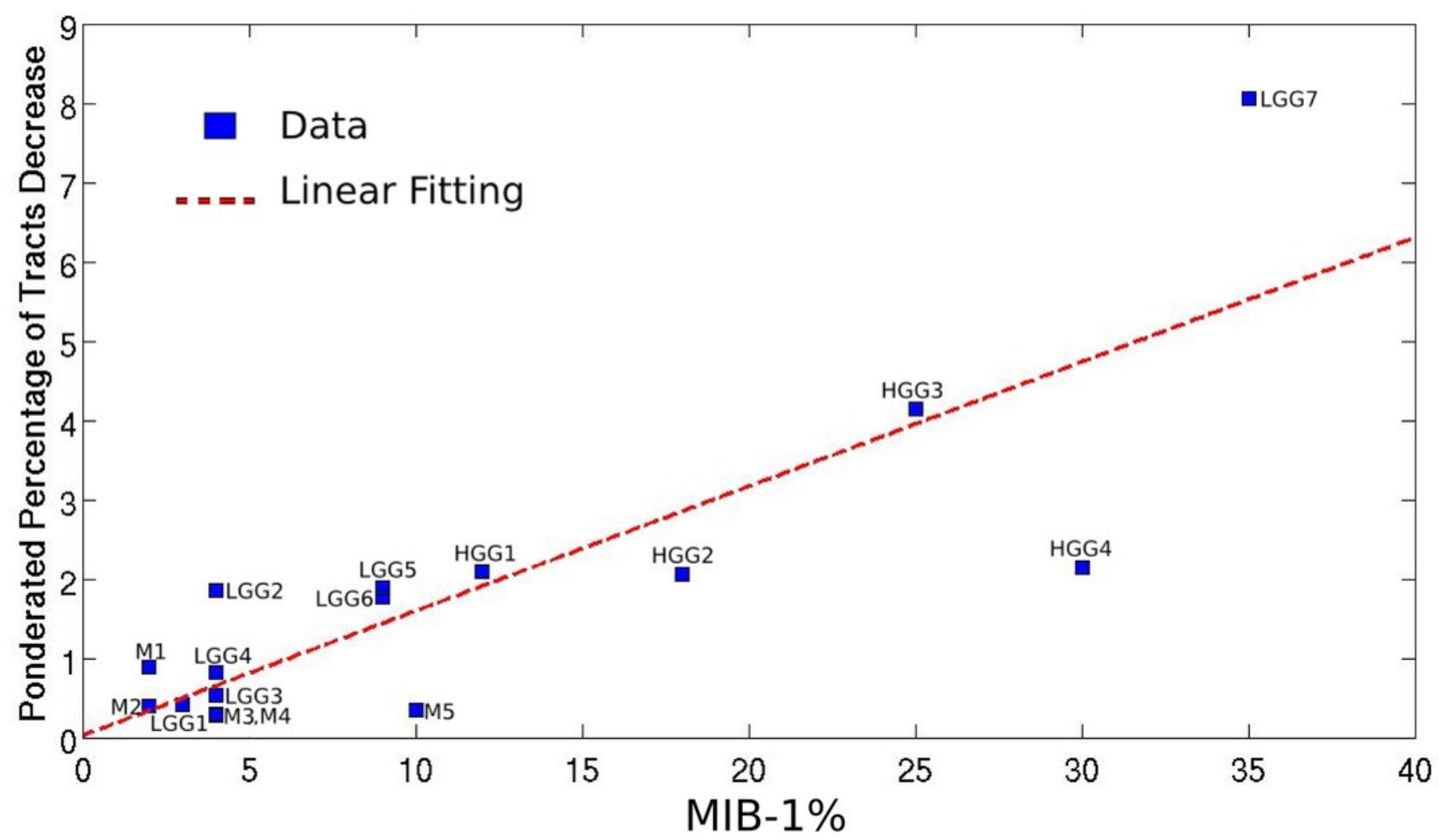




\section{Figure 3}

Figure 3 : Comparative tractography study between the two hemispheres in the five cases of meningioma.

Cases M1, M2 and M5: Estimation of the fiber tracts between the main target and the dilated region homologous to the tumor in the healthy hemisphere; comparison with the contralateral lesioned architecture. In the case M1 the ascending fibers from the thalamus to cerebral cortex and the descending fibers from the fronto-parietal cortex to subcortical nuclei and spinal cord were splayed into different branches in the lesioned hemisphere. The same alteration pattern was identified in the cortico-ponto-cerebellar tract reconstructed in patient M2, as a result of the tumor mass. The fiber tracts that leave the internal capsule ventrally and continue into the cerebral peduncles, pons and piramidal tract in the pathological hemispehere of patient M5 resulted bulky displaced, in relation to the contralateral WM architecture. Cases M3 and M4: Estimation of the fiber tracts between the two target regions in the healthy hemisphere, and comparison with the contralateral lesioned architecture. The lesion mass changed the location and organization of the inferior fronto-occipital fasciculus tracked in the healthy hemisphere in both patients M3 and M4. 


\section{Meningiomas}

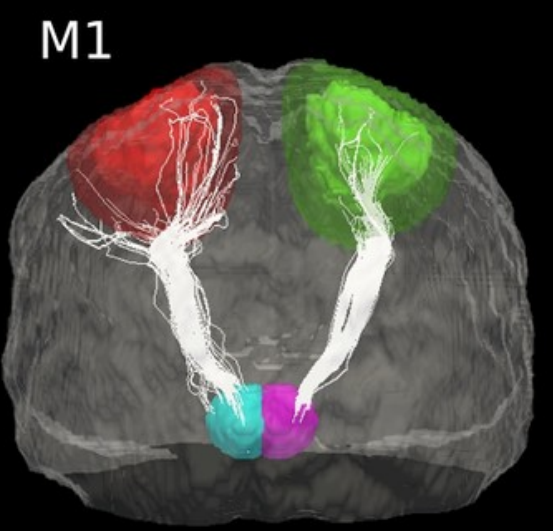

M2
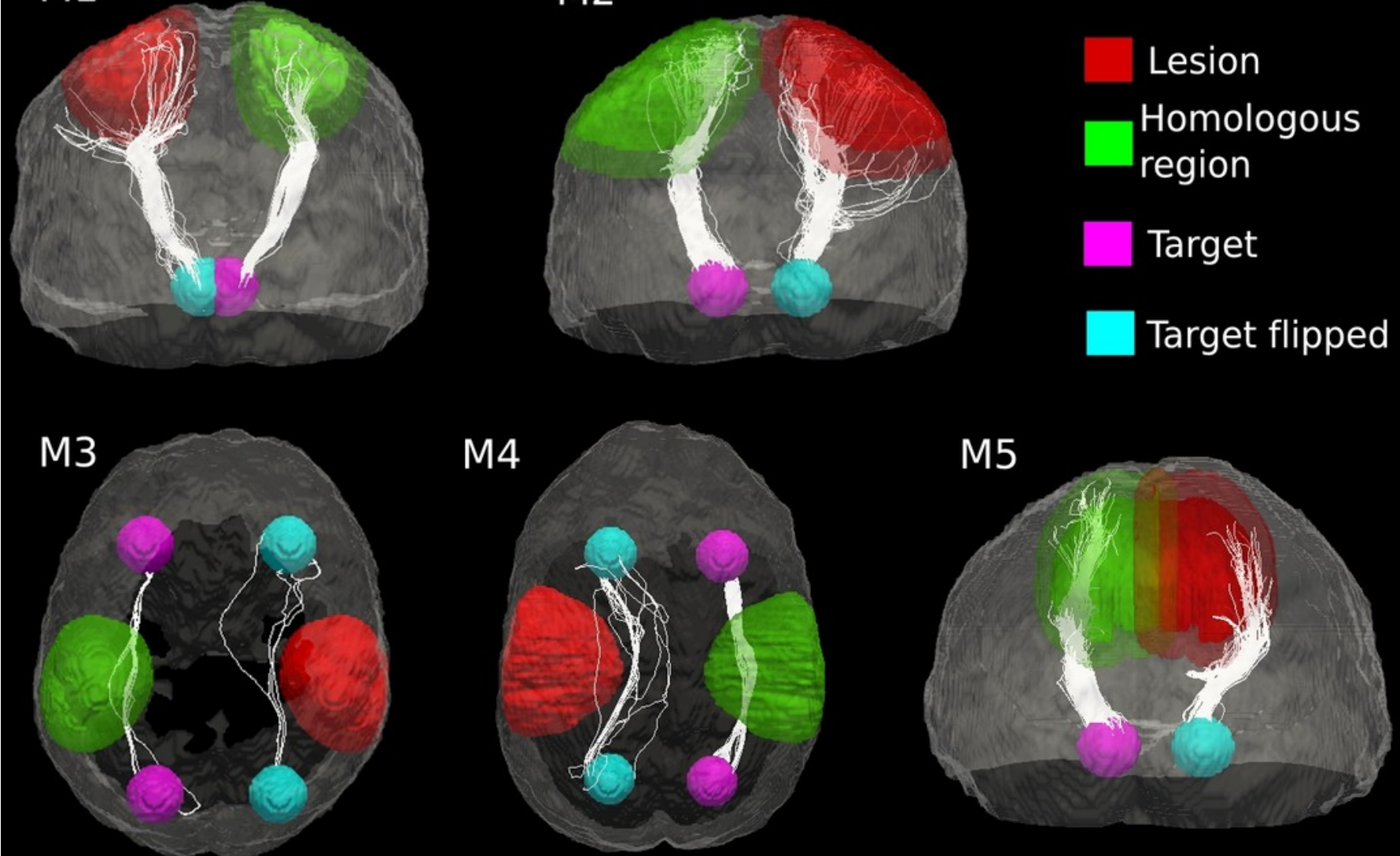

M4

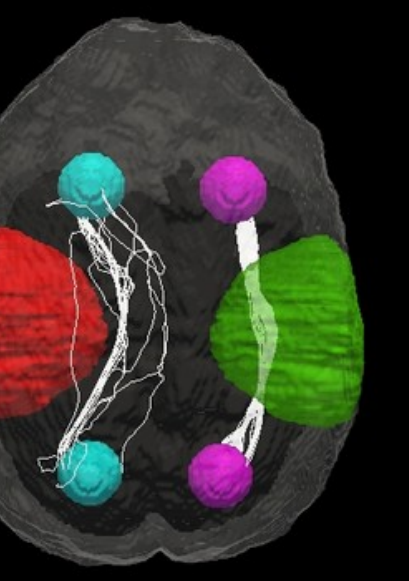

M5

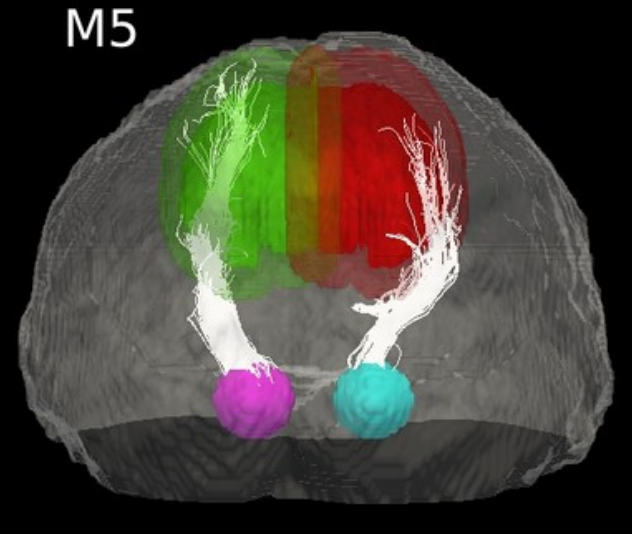




\section{Figure 4}

Figure 4: Comparative tractography study between the two hemispheres in the seven cases of low-grade glioma.

Cases LGG1 and LGG5: Estimation of the fiber tracts between the two target regions in the healthy hemisphere, and comparison with the contralateral affected architecture. The superior bundle of Cingulum, reconstructed in the homologous region, resulted dorsally displaced in the pathological hemisphere in case LGG1, while its inferior pathway was not identified at all in the lesioned area. The inferior Fronto-Occipital Fasciculus, tracked in patient LGG5, was displaced as well.

Cases LGG2, LGG3, LGG4, LGG6, and LGG7: estimation of the fiber tracts between the main target and the dilated region homologous to the tumor in the healthy hemisphere; comparison with the contralateral affected architecture. The Superior Longitudinal Fascicle identified in case LGG2 could not be tracked in the affected hemisphere. In patient LGG3, the inferior bundle of the Arcuate Fasciculus that connected the homologous region to the target $\mathrm{ROI}$ resulted destroyed in the pathological area. The Cerebellar tracts tracked in patients LGG4 and LGG6 appeared disrupted by the infiltrating tumor mass. Finally, in patient LGG7 the inferior part of Arcuate fasciculus, reconstructed in the healthy hemisphere resulted mostly destroyed in the contralateral area. 


\section{Low Grade Gliomas}

Lesion $\square$ Homologous region $\square$ Target $\square$ Target flipped LGG1

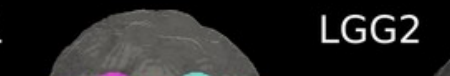

\section{LGG3}

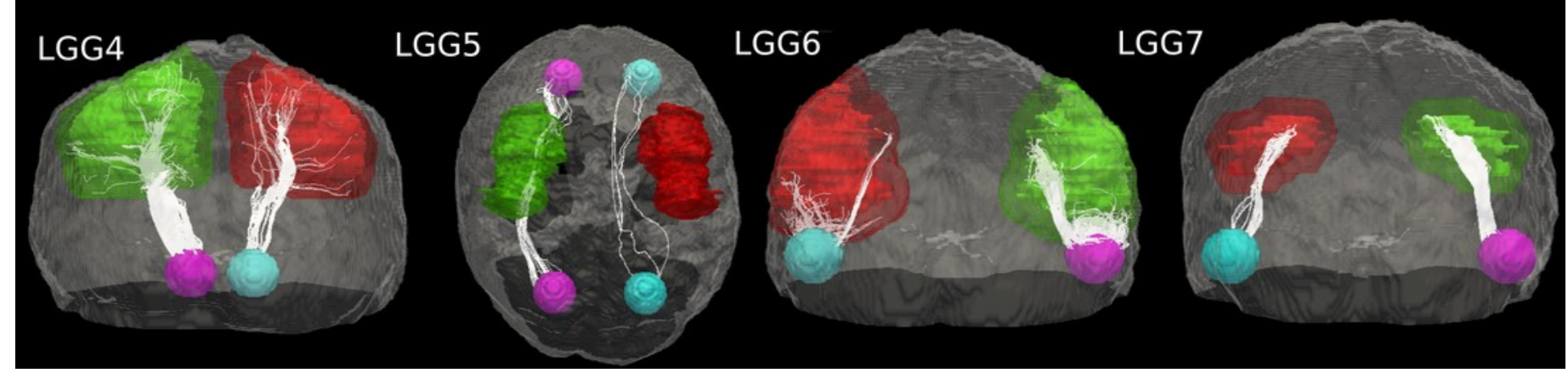




\section{Figure 5}

Figure 5: Comparative tractography study between the two hemispheres in the four cases of high-grade glioma.

Cases HGG1, HGG2, HGG3, and HGG4: estimation of the fiber tracts between the main target and the dilated region homologous to the tumor in the healthy hemisphere; comparison with the contralateral lesioned architecture. Tumor infiltration can be clearly seen in all the four cases. The Superior Longitudinal Fascicle in the affected hemisphere was largely disrupted in both patients HGG1 and HGG3. The Arcuate Fasciculus connecting the homologous area and the target ROI in the healthy hemisphere of case HGG2, resulted almost entirely destroyed in the tumor area. In addition, tractography in patient HGG4 demonstrated the huge loss of fiber tracts belonging to the Inferior Fronto-Occipital Fasciculus and the Cingulum reconstructed in the healthy brain contralateral area. 


\section{High Grade Gliomas}

\section{Lesion $\square$ Homologous region $\square$ Target $\square$ Target flipped}

HGG1

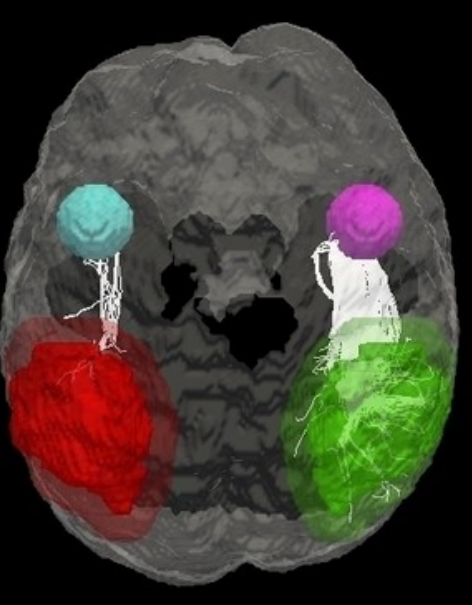

HGG3
HGG2

HGG4 


\section{Figure 6}

Figure 6: Connection probability maps in the two hemispheres for three exemplificative cases.

(Meningioma M3; Low-Grade Glioma LGG4; High-Grade Glioma HGG3). The probability maps, in which each voxel value corresponds to the number of streamlines that enter the voxel divided by the total number of streamlines in the input, are overlaid onto coronal and axial views of the b0 brain volume. Results showed different degrees of severity in the tumorinduced alterations of white matter tracts, as compared to the contralateral architecture. On the left and right sides, connectivity maps obtained by deterministic tractography confirm that the deterministic algorithm tracked all the important connections shown by the probabilistic analysis. 
PROBABILISTIC TRACTOGRAPHY

Healthy Hemisphere

Deterministic
Pathological Hemisphere

MENINGIOMA
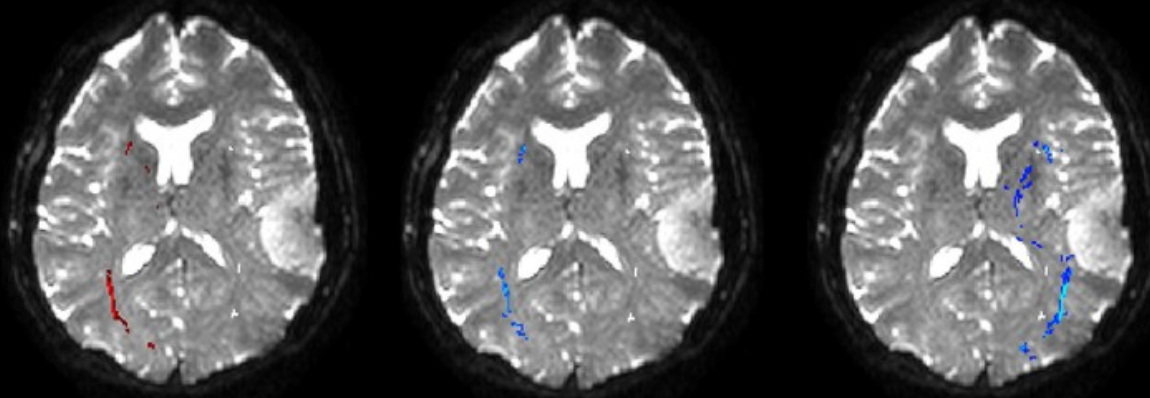

LOW-GRADE GLIOMA
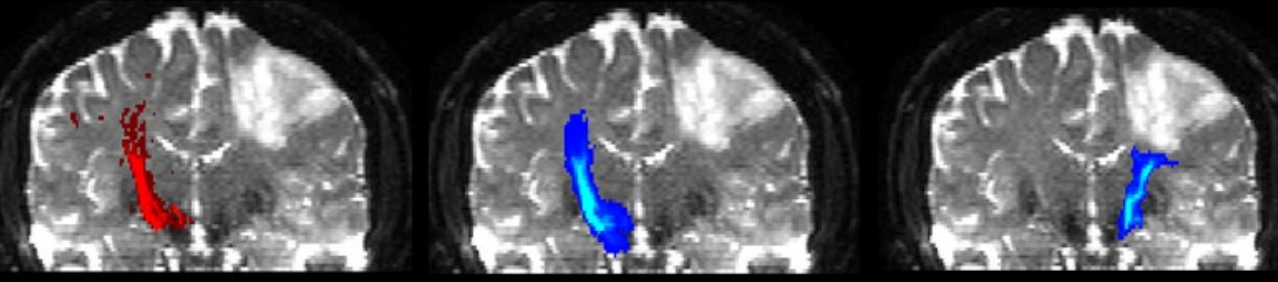

HIGH-GRADE GLIOMA

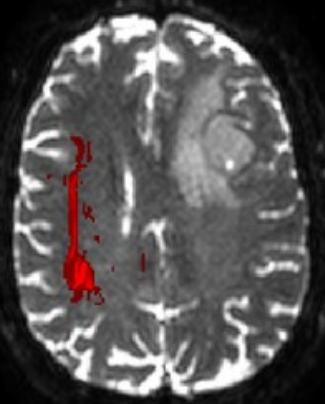

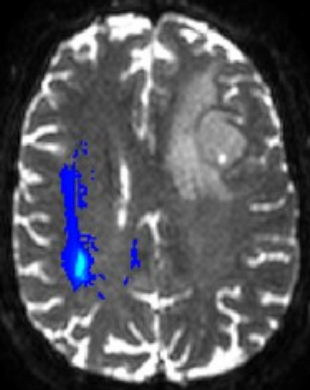

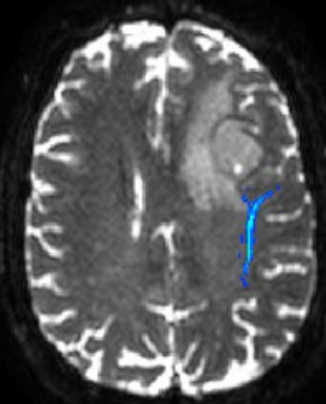

Deterministic

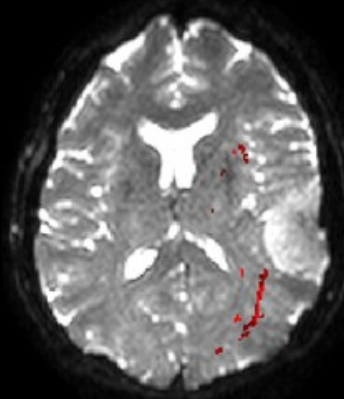

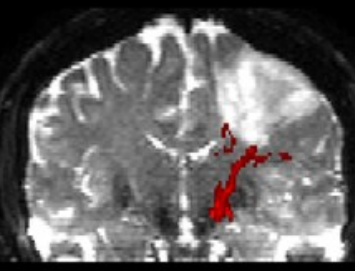

